\title{
Fractal Weyl law behavior in an open Hamiltonian system
}

\author{
Jordan A. Ramilowski, ${ }^{1,2}$ S. D. Prado, ${ }^{1,3}$ F. Borondo, ${ }^{1}$ and David Farrelly ${ }^{1,2}$ \\ ${ }^{1}$ Departamento de Química and Instituto Mixto de Ciencias Matemáticas CSIC-UAM-UC3M-UCM, Universidad Autónoma de Madrid, \\ Cantoblanco, 28049 Madrid, Spain \\ ${ }^{2}$ Department of Chemistry, Utah State University, Logan, Utah 84322-0300, USA \\ ${ }^{3}$ Instituto de Física, Universidade Federal do Rio Grande do Sul, P.O. Box 15051, 91501-970 Porto Alegre, RS, Brazil
}

(Received 26 March 2009; revised manuscript received 2 October 2009; published 30 November 2009)

\begin{abstract}
We numerically show fractal Weyl law behavior in an open Hamiltonian system that is described by a smooth potential and which supports numerous above-barrier resonances. This behavior holds even relatively far away from the classical limit. The complex resonance wave functions are found to be localized on the fractal classical repeller.
\end{abstract}

DOI: 10.1103/PhysRevE.80.055201

PACS number(s): 05.45.Mt, 03.65.Sq, 05.45.Df

The classical and quantum dynamics of open Hamiltonian systems are relevant to a variety of topics of current interest in macroscopic and microscopic physics. For example, in planetary physics, the formation of binaries in the Kuiper belt may have proceeded through the formation of transitory objects in chaotic layers of phase space trapped close to above-barrier Kolmogorov-Arnold-Moser (KAM) islands $[1-3]$. The analogs of these states in open quantum Hamiltonians are resonances (quasibound states) which, in general, are predicted to be localized on an object known as the classical repeller [4]. The repeller is the intersection of two fractal sets of classical trajectories one of which remains trapped in the infinite past and the other in the infinite future, denoted as $K_{-}$and $K_{+}$, respectively. The fractal nature of these sets has led to the prediction of a fractal Weyl law for flows in which the number of long-living quantum resonances scales as $\hbar^{-\left(1+d_{H}\right)}$, where $d_{H}$ is the partial Hausdorff dimension of the repeller [5].

In open maps the number of resonances has already been found to obey a similar fractal Weyl law, $\hbar^{-d}$, except that $d$ is now the partial fractal dimension of the trapped set. This relates to the original Weyl law [6] conceived for closed systems which states that the number of eigenstates up to energy $E$ which fits into the available phase-space volume of the classical system scales as $\hbar^{-d}$, with $d$ being the actual (integer) dimensionality of quantum space. In addition, in open maps the associated quantum Husimi distributions are observed to cling to the classical repeller $[7,8]$. Although the fractal Weyl law has been observed in particular maps such as the baker map and the kicked rotor [5,7-12], there have been few previous studies of this problem in open Hamiltonian systems [13] even though such systems are of direct physical interest; e.g., the chaotic ionization of hydrogen atom interacting with a circularly polarized microwave (CPM) field which exhibits above-barrier chaotic trapping [14].

Here we present an examination of above-barrier quantum resonance (Gamow) states in a model Hamiltonian whose classical dynamics is chaotic. The system is described by a smooth potential and numerous above-barrier resonances are supported. The model we use is chosen to capture essential features of the chaotic ionization dynamics of atoms in rotating fields; further, we propose, that this mechanism may also be important in complex formation in certain chemical reactions. We find that, not only does the fractal Weyl law hold for a typical (generic) open Hamiltonian system [15], but it also holds even in the vicinity of $\hbar=1$, i.e., far from the classical limit.

The investigation of a fractal law for open analytical Hamiltonian systems is problematic for a number of technical reasons, including the larger dimensionality of phase space $N$ needed to observe the chaotic repeller; unlike in unidimensional maps, for which $N=2$, in an autonomous Hamiltonian the repeller exists only if $N \geq 4$ which compounds the computational challenges involved. An additional computational difficulty is the calculation of resonance eigenfunctions in the limit $\hbar \rightarrow 0$ because of the attendant growth in the size of the basis needed to converge the calculations. Some of these obstacles have been overcome in a previous study which reported fractal Weyl law behavior in an open Hamiltonian whose potential-energy surface (PES) consisted of three Gaussian bumps [13]. However, for computational reasons only a rather limited number of resonances were included in the analysis and the structure of the resonance eigenstates themselves was not examined.

The model chosen here provides a realistic model of the chaotic ionization of atoms and of resonances in chemical reactions in that the PES features a potential well together with saddle points, and depending on the energy, the classical dynamics may be mixed (i.e., regular and chaotic) even above the saddle points. Computation of quantum complex (resonance) eigenvalues and quantum surfaces of section (QSOS) (see, e.g., Refs. [12,16]), based on Husimi distributions, reveals that the above-barrier resonance energies are localized on the classical repeller. For values of $\hbar$ away from the asymptotic limit there is progressively more delocalization. The advantage of the model used is that the calculations are more tractable than for, say, the $\mathrm{H}$ atom interacting with rotating fields for which the Coulomb term complicates the computations.

The model is a modification of the Hénon-Heiles (HH) Hamiltonian [17]

$$
H=\frac{1}{2}\left(p_{x}^{2}+p_{y}^{2}\right)+\frac{1}{2}\left(x^{2}+y^{2}\right)+\lambda\left(x^{2} y-\frac{1}{3} y^{3}\right)-\omega\left(x p_{y}-y p_{x}\right),
$$

where, throughout, $\lambda=0.1$ and $\omega=0.1$. The modification is the presence of a Coriolis term-the term in $\omega$-which is 


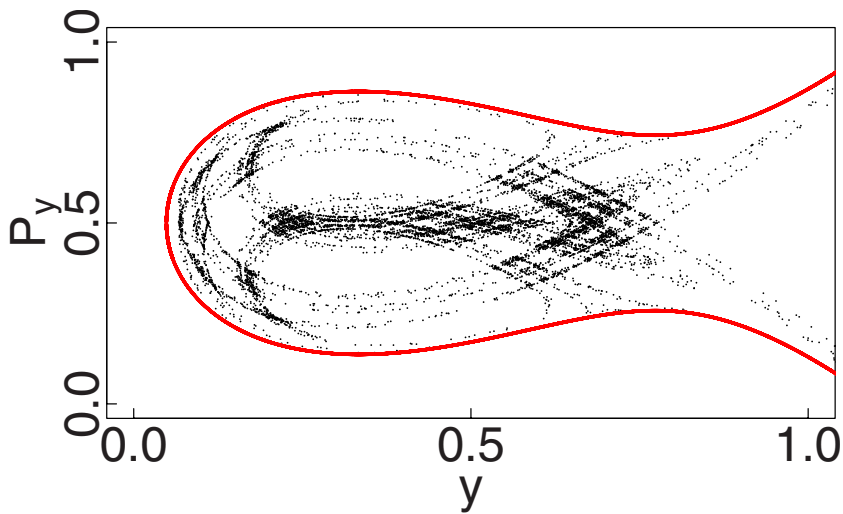

FIG. 1. (Color online) Superposition of the two branches of the repeller computed as described in the text and projected onto the SOS defined by $x=0, \dot{x}<0$ and $E=1.8 E_{s}$. The bounding curve, solid black line (red online), of the SOS is also shown. Both axes have been scaled to the interval $(0,1)$ to allow for comparison with the Husimi plot of Fig. 3.

designed to simulate the addition of, e.g., a CPM field or a magnetic field to a Rydberg atom [18]. Because the Hamiltonian does not have rotational symmetry this angularmomentum-like term is not a conserved quantity. Furthermore, the presence of the Coriolis term means that it is no longer possible to define a potential-energy surface-instead one can resort to using the device of a zero velocity surface (ZVS) (see, e.g., Ref. [14]). Finally, time reversal symmetry of the system is broken. Energies and widths are scaled by the energy of the three saddle points in the ZVS, i.e., $E_{s}=\left(1-\omega^{2}\right)^{3} / 6 \lambda^{2}=16.17165$.

The structure of the repeller is shown in Fig. 1 for $\omega=0.1$ at an energy rather high above the saddle-point energy. The repeller was computed by integrating trajectories forward and backward in time. The initial conditions of trajectories which survived (i.e., did not escape) for a time $\tau_{0}$ were saved. Survivors were then reintegrated for a time $20 \tau_{0}$ and their intersections with an appropriate Poincaré surface of section (SOS) were recorded. In this case the SOS chosen is defined by $x=0, \dot{x}<0$.

The effect of adding the Coriolis term is that, for nonzero $\omega$, relatively large KAM islands may co-exist with-but are not part of - the repeller for energies considerably above the energy of the saddle points. Here we do not consider resonances directly associated with these islands. However, the Coriolis terms has the effect of bringing out the structure of the repeller more clearly than for the pure HH system $(\omega=0$ [17]). Thus, varying $\omega$ allows for the fine tuning of the dynamics in the energy regime of interest.

The method of complex rotation was used to compute the complex resonance energies $E_{n}=E_{r}-i \Gamma_{n} / 2$, where $\Gamma_{n}$ is the resonance width [19]. This was accomplished by rotating the coordinates into the complex plane by an angle $\theta$, i.e., $q_{i} \rightarrow q_{i} e^{i \theta}$ and then diagonalizing the resulting Hamiltonian matrix in a two-dimensional isotropic oscillator basis $|n, m\rangle$ [17]. In principle the procedure is straightforward although care must be exercised to ensure that resonances are distinguished from scattering states. This can be accomplished by examining so-called $\theta$ trajectories, i.e., as the angle $\theta$ is var-
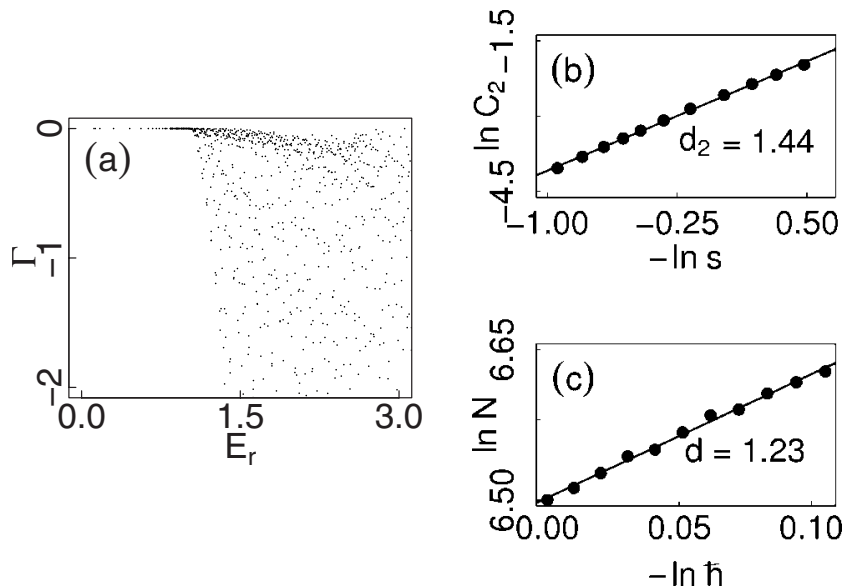

FIG. 2. Frame (a) shows resonance positions $\left(E_{r}\right)$ and widths ( $\Gamma$ ) (scaled by the saddle-point energy $E_{s}$ ) for $\hbar=1$. In (b) the classical quantity $\ln C_{2}(s)$ is shown vs $\ln s$ together with a best fit to a line whose slope is the correlation dimension [see Eq. (2)], i.e., $d_{2}=1.442 \pm 0.008$. Frame (c) shows a best fit to box counted quantum resonances with slope $d=1.231 \pm 0.028$. The quantum resonances scale as $\hbar^{-m / 2}$ or $\hbar^{-1.22}$ [5], where $m=\left(1+d_{2}\right)$ is the dimension of the trapped set; the quantum box counting dimension is 1.23. Despite considering a relatively narrow range of $\hbar$, lying far above the classical limit, the classical and quantum fractal dimensions agree well.

ied the resonances, as distinct from scattering states, converge. A large number of resonances lying above the saddle points are required to achieve the quality of statistics needed to determine how the number of resonances scales with $\hbar$.

The complex energy spectrum for the Hamiltonian of Eq. (1) contains resonances lying below as well as above the saddle points. Subsaddle states decay by tunneling. However, the states of interest lie above the saddle points and, therefore, a large basis set must be used to converge these resonances. As $\hbar$ is decreased the number of states below the saddles grows, and therefore, the size of the basis must be increased. For this reason it is difficult to access the very small values of $\hbar$ or equivalently, the very high-lying states-for which it is normally assumed that the fractal Weyl law will hold. Working on the observation that, in general, asymptotic expansions often provide good agreement even outside their strict domains of validity, we examined resonance statistics for $\hbar$ in the vicinity of $\hbar=1$. Numerically, resonances were computed by direct diagonalization and also, as check, by using the Arnoldi method which takes advantage of the sparsity of the Hamiltonian matrix [13]. The Arnoldi method has the twin merits that (i) a larger basis can be employed and (ii) it allows access to selected portions of the spectrum. However, only a relatively small subset of resonances can be computed in this way [20].

Distributions of resonances in the range $0.9 \leq \hbar \leq 1$ were then computed. Figure 2 shows the complex resonance eigenvalues obtained for $\hbar=1$. By counting the number of states, $N(\hbar)$, in eight different rectangular boxes of size $(1,1.24 \hbar)$ located around $E_{r}=1.8 E_{s}$ and averaging over these sets of data we were able to establish that the number of states follow a Weyl law with $d=1.231 \pm 0.028$ (see lower inset in Fig. 2). Similarly, and also as shown in the figure, the 
dimension of the classical repeller of Fig. 1 computed from the Poincaré map is fractal with correlation dimension $d_{2}=1.442 \pm 0.008$, where $[21]$

$$
d_{2}=\lim _{s \rightarrow 0} \frac{\ln C_{2}(s)}{\ln s}
$$

Here $s$ is the edge length of an $n$-dimensional cube and $C_{2}(s)$ is the correlation sum [21]

$$
C_{2}(s)=-\lim _{M \rightarrow \infty} \frac{1}{M^{2}} \sum_{k, \ell=1}^{M} \Theta\left(s-\left\|\mathbf{q}_{k}^{n}-\mathbf{q}_{\ell}^{n}\right\|\right),
$$

where $M$ is the number of points in the repeller, $\Theta$ is the Heaviside step function, and $\mathbf{q}_{k}$ are the points of the repeller. The fractal dimension, $m$, is related to the correlation dimension computed from a Poincaré map as $m=1+d_{2}$ [21]. Using the classical data we found that $d_{2}=1.442 \pm 0.008$ which leads to a fractal dimension for the repeller of $m=2.44$.

According to Ref. [5] the quantum resonances in an energy interval should scale as $\hbar^{-m / 2}$, where $m$ is the dimension of the trapped set for the energies in that interval. This is in excellent agreement with quantum box counting since $m / 2=1.22$ while the quantum box counting gives $d=1.23$. This prediction is borne out remarkably well by Fig. 2 and is an illustration of the fractal Weyl law in an open Hamiltonian system (rather than in a map). The number of resonances in the boxes varied from 686 to 827 depending on $\hbar$.

In addition to the conjecture that for generic open systems fractal Weyl law behavior will be observed, it is also expected that Husimi functions will coagulate onto fractal sets (i.e., onto the repeller) in the limit $\hbar \rightarrow 0$ [7]. For finite $\hbar$ Husimi functions will not truly be confined to fractal sets and will appear somewhat blurred due to quantum effects. However, as $\hbar$ is decreased then classical structures, on progressively finer scales, will become apparent in the Husimi functions. The $\hbar \rightarrow 0$ limit is itself of physical interest in that this limit corresponds, e.g., to the ionization of ultrahigh Rydberg states.

Because the Hamiltonian is complex and non-Hermitian the left, $\Psi_{L}^{(i)}$, and right, $\Psi_{R}^{(i)}$, eigenfunctions do not satisfy the usual (Hermitian) identity $\Psi_{L}^{(i)}=\Psi_{R}^{(i) *}$ and, consequently, $\rho_{i}=\Psi_{L}^{(i)} \Psi_{R}^{(i) *}$ is a complex quantity. In fact, observables are associated with neither $\Psi_{L}^{(i)}$ nor $\Psi_{R}^{(i)}$ but with $\sqrt{\Psi_{L}^{(i)} \Psi_{R}^{(i)}}[22]$. This complicates the computation of Husimi distributions as has been discussed by Buchleitner, et al. [23] who pointed out that Husimi distributions for individual complex eigenstates have the peculiar property that they can be negative and a sum needs to be made, in principle, over all complex energies [23]. For this reason and, in analogy with previous computations in quantum maps, Husimi distributions are averaged over an energy range of finite width. We use the following definition of the averaged Husimi function whose derivation includes both left and right eigenstates and which is in the spirit of Bogomolny $[7,23,24]$

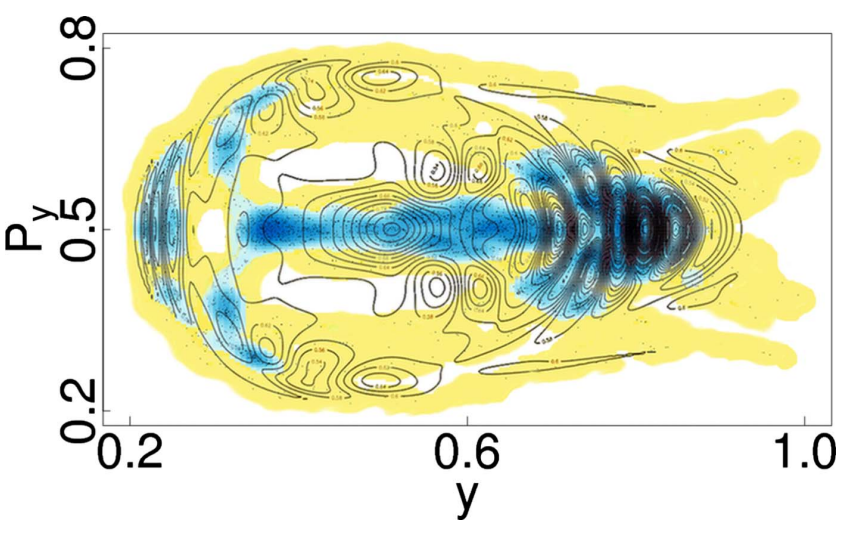

FIG. 3. (Color online) Contours of the averaged Husimi function calculated as described in the text superimposed on a representation of the classical repeller. Twenty resonance states on each side of $E=1.8 E_{s}$ were included. The points which represent the classical repeller on the SOS have been kernel smoothed which, in essence, assigns a local density of points and then colors that section of the plot accordingly [3] — compare to Fig. 1. The color scale runs from white through light gray (yellow and light blue online) to dark gray (dark blue online) and represents the density from low to high accordingly.

$$
\left|\left\langle\Omega \mid \phi_{E}\right\rangle\right|^{2}=\frac{1}{\pi} \operatorname{Im} \sum_{i} \frac{\left\langle\bar{\Psi}_{L}^{(i)}|R(\theta)| \Omega\right\rangle\left\langle\bar{\Psi}_{L}^{(i)}|R(\theta)| \bar{\Omega}\right\rangle}{E_{i \theta}-E} .
$$

Here $\phi_{E}$ represents the probability amplitude at real energy $E$ and $|\Omega\rangle$ is a coherent state; $\Psi_{L}$ is a complex rotated eigenstate expressible in terms of the isotropic oscillator basis vectors; $E_{i \theta}$ is the complex energy of the eigenstate and the overbar notation signifies, e.g., that $\langle\bar{\Psi}|$ is the complex conjugate of $\langle\Psi| ; R(\theta)$ is the complex rotation operator [23]. Very recently, Ermann et al. proposed a different, although related phase-space representation for open quantum systems [12].

For narrow resonances simplifications of Eq. (4) are possible. By projecting the states onto a basis of isotropic oscillator functions one avoids computing basis vectors in the complex coordinate plane, a procedure which is numerically unstable since basis vectors which are oscillatory along the real axis may diverge exponentially in the complex plane [23]. In this case one then needs to compute matrix elements of $R(\theta)$ in the basis used although these matrix elements themselves ultimately diverge - the resonance eigenfunctions are not $L^{2}$ functions.

We adopted the following procedure to project the fourdimensional Husimi distribution onto a two-dimensional hypersurface in phase space so as to generate a QSOS. A narrow interval of energy was selected around some energy of interest $E_{0}$. Equation (4) was then used with the resonance eigenstates projected onto the isotropic oscillator basis. Only resonance states with widths smaller than some width, $\Gamma_{0}$, were included in the summation and the matrix elements $\langle n|R(\theta)| m\rangle$ were approximated by their lowest order (i.e., diagonal) expansion in $\theta$. For states with narrow widths, as is the case here, this is an excellent approximation. The QSOS was then computed by fixing $x=0$ and computing the conju- 
gate momentum $p_{x}$ using the classical Hamiltonian at energy $E$. Because the boundary of the classically allowed region itself changes with energy this procedure is not entirely satisfactory when computing an average Husimi QSOS. However, provided that the energy range is kept sufficiently small the errors so introduced are expected to be minimal and this was verified by direct computation.

Figure 3 shows a typical example of a QSOS computed in this way. The averaged Husimi distribution is clearly localized on the fractal repeller sets $K_{+}$and $K_{-}$which are also shown in the figure. However, this tendency for the Husimi to coagulate onto the repeller is mitigated by the relatively large value of $\hbar=1$ used in constructing the figure. The $\mathrm{Hu}-$ simi distribution is somewhat delocalized over the repeller and does not precisely follow the contours of the fine scale classical structures. It is also apparent in Fig. 3 that the quantum density builds up close to the saddle point. The reason for this is that the quantum particle senses the presence of classical turning points in the complex plane and, therefore, slows down which leads to a buildup in probability density in the vicinity of the saddle point. This is consistent with the recent findings of Keating et al. who note that for longer living states, the long lifetime allows interference and diffraction effects to accumulate thereby washing out the fractal structure to some extent [11].

In summary: the fractal Weyl law was found to hold in an open Hamiltonian system. Despite working far from the asymptotic limit $\hbar \rightarrow 0$ the resonance energies manifested clear fractal behavior and averaged Husimi distributions reflected rather faithfully the structure of the classical repeller.

We acknowledge support from the Ministerio de Educación y Ciencia (Spain) [Projects No. MTM2006-15533, No. MTM2009-14621, and CONSOLIDER 2006-32 (iMath)]; the Comunidad de Madrid [Project No. S-0505/ESP0158 (SIMUMAT)]; a grant (to S.D.P.) from NanoforumEULA (support action funded by the European Union), and the NSF (USA) through Grant No. CHE-0718547.
[1] A. D. Perry and S. D. Wiggins, Physica D 71, 102 (1994).

[2] S. A. Astakhov, A. D. Burbanks, S. Wiggins, and D. Farrelly, Nature (London) 423, 264 (2003).

[3] E. A. Lee, S. A. Astakhov, and D. Farrelly, Mon. Not. R. Astron. Soc. 379, 229 (2007).

[4] P. Gaspard, Chaos, Scattering, and Statistical Mechanics (Cambridge University Press, Cambridge, England, 1998).

[5] W. T. Lu, S. Sridhar, and M. Zworski, Phys. Rev. Lett. 91, 154101 (2003).

[6] H. P. Baltes and E. R. Hilf, Spectra of Finite Systems (B-I Wissenschaftsverlag, Mannheim, 1978).

[7] J. P. Keating, M. Novaes, S. D. Prado, and M. Sieber, Phys. Rev. Lett. 97, 150406 (2006).

[8] D. L. Shepelyansky, Phys. Rev. E 77, 015202(R) (2008).

[9] H. Schomerus and J. Tworzydlo, Phys. Rev. Lett. 93, 154102 (2004).

[10] S. Nonnenmacher, Lectures Notes in Physics (Springer, Berlin, 2006), Vol. 690.

[11] J. P. Keating, S. Nonnenmacher, M. Novaes, and M. Sieber, Nonlinearity 21, 2591 (2008).

[12] L. Ermann, G. G. Carlo, and M. Saraceno, Phys. Rev. Lett.
103, 054102 (2009)

[13] K. Lin, J. Comput. Phys. 176, 295 (2002).

[14] A. F. Brunello, T. Uzer, and D. Farrelly, Phys. Rev. A 55, 3730 (1997).

[15] M. Kopp and H. Schomerus, e-print arXiv:0906.5320.

[16] P. A. Dando and T. S. Monteiro, J. Phys. B 27, 2681 (1994).

[17] J. Kaidel, P. Winkler, and M. Brack, Phys. Rev. E 70, 066208 (2004).

[18] A. Buchleitner, D. Delande, and J. Zakrzewski, Phys. Rep. 368, 409 (2002).

[19] Y. K. Ho, Phys. Rep. 99, 1 (1983).

[20] R. Barrett, M. Berry, T. F. Chan, J. Demmel, J. Donato, J. Dongarra, V. Eijkout, R. Pozo, C. Romine, and H. Van der Vorst, Templates for the Solution of Linear Systems: Building Blocks for Iterative Methods (SIAM, Philadelphia, PA, 1994).

[21] W. Lauterborn and U. Parlitz, J. Acoust. Soc. Am. 84, 1975 (1988).

[22] H. Barkay and N. Moiseyev, Phys. Rev. A 64, 044702 (2001).

[23] A. Buchleitner, B. Grémaud, and D. Delande, J. Phys. B 27, 2663 (1994).

[24] E. B. Bogomolny, Physica D 31, 169 (1988). 